Revista Brasileira de Meteorologia, v.29, n.2, 197 - 208, 2014

\title{
EVENTOS EXTREMOS DE PRECIPITAÇÃO NAS MESORREGIÕES DA PARAÍBA E SUAS RELAÇÕES COM A TSM DOS OCEANOS TROPICAIS
}

\author{
JOSÉ NILDO DA NÓBREGA ${ }^{1}$, CARLOS A. C. DOS SANTOS ${ }^{1}$, OSEAS M. GOMES ${ }^{2}$, BERGSON G. \\ BEZERRA $^{3}$, JOSÉ IVALDO B. DE BRITO ${ }^{1}$ \\ ${ }^{1}$ Universidade Federal de Campina Grande (UFCG), Unidade Acadêmica de Ciências Atmosféricas, \\ Campina Grande, PB, Brasil. \\ ${ }^{2}$ Universidade Estadual da Paraíba (UEPB), Departamento de Estatística, Campina Grande, PB, Brasil. \\ ${ }^{3}$ Universidade Federal do Rio Grande do Norte (UFRN), Natal, RN, Brasil.
}

nildo_nobrega@yahoo.com.br, carlos@dca.ufcg.edu.br,oseasmachado@gmail.com.br, bergson@ccet.ufrn.br, ivaldo@ dca.ufcg.edu.br

Recebido Junho de 2012 - Aceito Julho de 2013

\begin{abstract}
RESUMO
O objetivo desse estudo foi identificar tendências de índices extremos de precipitação sobre a Paraíba, e correlacioná-los com as anomalias de Temperatura da Superfície do Mar (TSM) dos Oceanos Atlântico e Pacífico. Os dados de precipitação diária, no período de 1935 a 2004, foram oriundos de 23 postos pluviométricos pertencentes ao Instituto Nacional de Meteorologia (INMET). Utilizou-se o programa RClimdex para calcular os índices extremos de precipitação, e aplicou-se o método dos mínimos quadrados e o teste não paramétrico de Mann-Kendall para identificar as tendências e suas respectivas significâncias estatísticas. Em seguida, foram calculadas as correlações entre os índices extremos de precipitação e a TSM. Foi encontrado maior número de localidades com tendências positivas no período de 1935 e 1969, e as maiores correlações foram com o Atlântico. As Mesorregiões do Sertão e Agreste do estado da Paraíba tiveram relação com as anomalias de TSM do Atlântico, Pacifico e Oscilação Multidecadal do Atlântico (OMA), a Borborema com as do Atlântico e OMA e, o Litoral com o Atlântico.
\end{abstract}

Palavras-Chave: Semiárido, ZCIT, Mann-Kendal, RClimdex, Oceano Atlântico, Oceano Pacífico.

ABSTRACT: EXTREME PRECIPITATION EVENTS IN THE MESOREGIONS OF PARAÍBA AND ITS RELATIONSHIP WITH THE TROPICAL OCEANS SST

The aim of this study was to identify trends in extreme precipitation indices on the Paraíba State, and to correlate them with the Sea Surface Temperature (SST) anomalies in the Atlantic and Pacific Oceans. The daily precipitation data for the period 1935-2004, were from 23 rain gauges belonging to Instituto Nacional de Meteorologia (INMET). To calculate the extreme rainfall indexes the RClimdex software was used. Then, the statistical least squares method and the non-parametric Mann-Kendall test were applied to identify trends and their statistical significance. The correlation between the extreme precipitation indices and the SST were then calculated. It was found a higher number of sites with positive trends in the period 1935 to 1969, and the highest correlations were with the Atlantic. The Sertão and Agreste Mesoregions of Paraiba state were well related to SST anomalies in the Atlantic, Pacific and Atlantic Multidecadal Oscillation (AMO), Borborema with the AMO and the Atlantic and the Litoral with Atlantic.

Keywords: Semiarid, ITCZ, Mann-Kendal, RClimdex, Atlantic Ocean, Pacific Ocean. 


\section{INTRODUÇÃO}

Durante as últimas décadas têm sido estudado as variabilidades e/ou tendências dos índices extremos de precipitação no Nordeste do Brasil (NEB), bem como a sua relação com as Temperaturas da Superfície do Mar (TSM). O NEB é uma região cujo clima predominante é semiárido, que apresenta substanciais variações temporais e espaciais da precipitação pluvial, e elevadas temperaturas ao longo do ano. A parte norte do NEB, onde se localiza o estado da Paraíba, tem sua curta estação chuvosa dependente do comportamento das TSM dos Oceanos Atlântico (Dipolo) e Pacífico Equatorial (El Niño/La Niña) (Hastenrath, 2012).

Santos e Manzi (2011) afirmam que as possíveis mudanças climáticas poderão acarretar significativos impactos nos setores natural, social e econômico, assim como, os extremos do clima associados à precipitação poderão também afetar o consumo de energia, o conforto humano e o turismo (Santos et al., 2009). Kostopoulo e Jones (2005) afirmam que o aumento nas perdas econômicas devido a extremos de tempo e, especialmente, o aumento na perda de vidas tem sido regularmente notícias de jornais, despertando o interesse da comunidade científica em estudar os fenômenos climáticos associados.

Eventos extremos de precipitação estão sendo estudados em escala global, nacional e regional. Em escala global, análises mais compreensivas dos extremos climáticos foram discutidas no Quarto Relatório de Avaliação do IPCC (IPCC, 2007). Na escala nacional e regional, estão sendo desenvolvidos estudos das mudanças e seus impactos na Ásia, Pacífico Sul, Caribe, África, América do Sul e América do Norte. Existem marcantes consistências entre os resultados obtidos por estes estudos em termos de temperatura, porém com menos coerência espacial nos extremos de precipitação (You et al., 2010; Santos et al., 2011). No Brasil, estudos com essa temática, como os de Santos e Brito (2007), mostraram tendências de aumento da precipitação total anual nos estados da Paraíba e Rio Grande do Norte, como também, em algumas regiões com aumento na ocorrência de chuvas superiores a $50 \mathrm{~mm}$. Santos et al. (2009), ao estudar tendências dos índices de precipitação total e eventos extremos de chuva no estado de Ceará, concluíram que apenas ocorreram mudanças locais de precipitação. Santos e Manzi (2011) mostraram que os índices extremos de precipitação, para a região sul do Ceará, apresentam características semelhantes às apresentadas para a região norte do estado. Entretanto, o comportamento dos dias consecutivos chuvosos, nessa parte do Ceará, indica que o período chuvoso é governado por outros sistemas atmosféricos, necessitando, portanto, aprofundar o estudo desse comportamento para obter um melhor entendimento dos fatores que afetam a precipitação sobre a região.
Os estudos, anteriormente descritos, corroborando com resultados obtidos por Aguilar et al. (2009) que afirmaram que mudanças na temperatura do ar e nos extremos de precipitação vêm ocorrendo na America Central e Norte da América do Sul ao longo das últimas décadas. Por outro lado, Haylock et al. (2006) analisando os dados das estações meteorológicas do Norte e Nordeste do Brasil não observaram um sinal de tendência consistente nos índices de precipitação para o NEB. Alguns outros estudos mostram que essas tendências estão relacionadas com a TSM.

Ao relacionar a precipitação do leste do NEB e a temperatura dos oceanos, Moura et al. (2009) afirmaram que a maior correlação observada é entre a precipitação e a TSM do Atlântico, do que com a do Pacífico. Eles afirmaram ainda que as anomalias positivas de TSM no Atlântico Tropical Sul induzem chuvas sobre o leste do NEB, já anomalias positivas no Pacífico Tropical inibem a chuva no leste do NEB.

Como a Paraíba fica situada a leste do NEB e é o estado da Região que apresenta uma das maiores variabilidades espacial no campo precipitação, assim, o objetivo principal deste trabalho é identificar possíveis tendências de índices extremos de precipitação sobre as Mesorregiões do estado da Paraíba, para o período de 1935 a 2004, subdivididos em dois períodos de 1935 a 1969 e 1970 a 2004, assim como, correlacionar os índices extremos de precipitação com anomalias de TSM nos oceanos Atlântico e Pacífico, e também, com a Oscilação Decadal do Pacífico (ODP) e a Oscilação Multidecadal do Atlântico (OMA). Sendo que, o período para as correlações entre os índices as anomalias de TSM são para o período de 1950 a 2004 já que não há dados observacionais de TSM antes de 1950.

\section{MATERIAL E MÉTODOS}

\subsection{Material}

A área estudada corresponde ao estado da Paraíba (Figura 1). Utilizaram-se dados diários de precipitação pluviométrica de 23 localidades, as quais estão apresentadas na Figura 1b e na Tabela 1, com boa representação espacial. Os dados foram oriundos da Superintendência de Desenvolvimento do Nordeste (SUDENE) e do Instituto Nacional de Meteorologia (INMET), adquiridos através da Unidade Acadêmica de Ciências Atmosféricas da Universidade Federal de Campina Grande (UACA/UFCG), para o período de 1935 a 2004, o qual foi dividido em dois períodos 1935 - 1969 e 1970 - 2004. Os dados de anomalias de TSM, para o período de 1950 a 2004, nas regiões previamente definidas, foram obtidos do site da National Oceanic and Atmospheric Administration (NOAA) (http://www. esrl.noaa.gov/psd/data/climateindices/list/). 

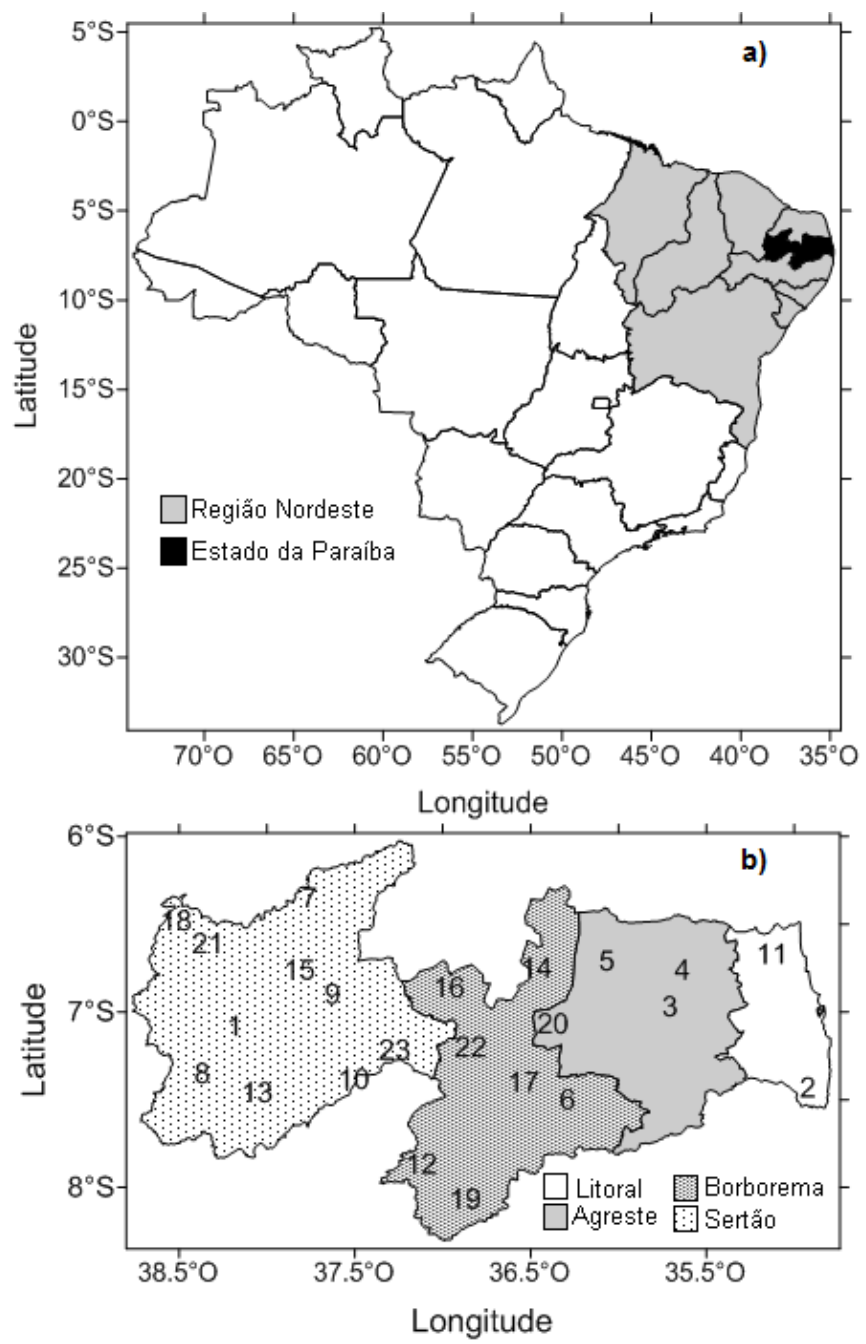

Figura 1 - a) Mapa do Brasil com destaque para a Região Nordeste e o Estado da Paraíba e b) mapa do estado da Paraíba apresentando as Mesorregiões e a distribuição espacial dos 23 postos pluviométricos utilizadas nesse estudo.

\subsection{Metodologia}

O programa RClimdex é baseado na linguagem R com saídas de tabelas e figuras, e tem capacidade de calcular 27 índices básicos recomendados pelo Variability and predictability of the ocean-atmosphere system (CLIVAR) do Climate Change Action Monitoring and Índices (ETCCDMI), bem como outros índices de temperatura e precipitação (Zhang e Yang, 2004). $\mathrm{O}$ mesmo tem sido bem utilizado em vários estudos, como os de Haylock et al., (2006) para América do Sul, Santos e Brito (2007) para o Semiárido Brasileiro e Santos et al. (2011) para Utah, Estados Unidos da América, e é adquirido de forma gratuita através de download no portal dos índices de ETCCDMI (http://cccma.seos.uvic.ca/ETCCDMI/) e disponível também na UACA/UFCG.

Tabela 1 - Postos pluviométricos utilizados na análise de possíveis tendências de índices extremos de precipitação no estado da Paraíba.

\begin{tabular}{|c|c|c|c|}
\hline ID & Localidade & $\begin{array}{c}\text { Latitude } \\
\left({ }^{\circ} \mathrm{S}\right)\end{array}$ & $\begin{array}{c}\text { Longitude } \\
\left({ }^{\circ} \mathrm{O}\right)\end{array}$ \\
\hline 1 & Aguiar & 7.08 & 38.18 \\
\hline 2 & Alhandra & 7.43 & 34.92 \\
\hline 3 & Areia & 6.97 & 35.70 \\
\hline 4 & Bananeiras & 6.76 & 35.63 \\
\hline 5 & Barra de Santa Rosa & 6.71 & 36.06 \\
\hline 6 & Cabaceiras & 7.50 & 36.28 \\
\hline 7 & Catolé do Rocha & 6.35 & 37.75 \\
\hline 8 & Conceição & 7.35 & 38.36 \\
\hline 9 & Condado & 6.90 & 37.62 \\
\hline 10 & Imaculada & 7.38 & 37.50 \\
\hline 11 & Mamanguape & 6.67 & 35.12 \\
\hline 12 & Monteiro & 7.87 & 37.12 \\
\hline 13 & Nova Olinda & 7.46 & 38.05 \\
\hline 14 & Pedra Lavrada & 6.75 & 36.46 \\
\hline 15 & Pombal & 6.76 & 37.81 \\
\hline 16 & Santa Luzia & 6.86 & 36.96 \\
\hline 17 & São João do Cariri & 7.40 & 36.53 \\
\hline 18 & São João do Rio do Peixe & 6.48 & 38.51 \\
\hline 19 & São João do Tigre & 8.07 & 36.87 \\
\hline 20 & Soledade & 7.07 & 36.37 \\
\hline 21 & Souza & 6.61 & 38.33 \\
\hline 22 & Taperoá & 7.20 & 36.83 \\
\hline 23 & Teixeira & 7.22 & 37.27 \\
\hline
\end{tabular}

Os oito índices de extremos climáticos, derivados de dados de precipitação diária, utilizados nessa pesquisa, foram: 1) Rx1dia (precipitação máxima em 1 dia); 2) Rx5dias (precipitação máxima em 5 dias consecutivos); 3) R10mm (número de dias em um ano com precipitação $\geq 10 \mathrm{~mm} / \mathrm{dia}$ ); 4) R30mm (número de dias em um ano com precipitação $\geq$ $30 \mathrm{~mm} / \mathrm{dia}$ ); 5) DCS (máximo número de dias consecutivos secos, ou seja, com precipitação total diária $\mathrm{PRCP}<1,0 \mathrm{~mm} /$ dia); 6) DCU (máximo número de dias consecutivos úmidos, ou seja, com PRCP $\geq 1,0 \mathrm{~mm} /$ dia); 7) SDII (índice simples de intensidade diária de precipitação); e 8) PRCPTOT (precipitação total anual). $\mathrm{Na}$ avaliação da homogeneização dos dados e no cálculo dos índices foi utilizada a metodologia de Zhang et al. (2005) implementada no software RClimdex. O controle de qualidade, que é um pré-requisito para a obtenção dos índices 
de extremos climáticos, consistiu na identificação de erros nos dados, conforme descrito por Viney e Bates (2004).

O software fornece dados estatísticos, para todos os índices, tais como: tendência linear anual calculada pelo método de mínimos quadrados; nível de significância estatística da tendência (valor $\mathrm{p}$ ); coeficiente de determinação $\left(\mathrm{r}^{2}\right)$ e erro padrão de estimativa; assim como os gráficos das séries anuais. Como os dados não se ajustaram a uma distribuição de frequência normal, foi utilizado o teste não-paramétrico de Mann-Kendall (Sneyers, 1990) para identificar se as tendências apresentam nível de significância para o intervalo definido. Nesse estudo só foram consideradas significativas às tendências, que apresentam nível de significância igual ou superior a $90 \%$ $(\mathrm{p} \leq 0,1)$, conforme mostrado nas Tabelas 2 e 3 .

O método de correlação de Pearson foi utilizado na análise das relações existentes entre as anomalias anuais de TSM dos Oceanos Pacífico (regiões de Niño 1+2, Niño 3, Niño 3.4 e Niño 4) e Atlântico (TNAI - Tropical North Atlantic Index e TSAI - Tropical South Atlantic Index) (Menezes et al., 2008), no período de 1950 a 2004 (período que dispunha de dados de TSM), e os índices de extremos climáticos anuais, que melhor representam a climatologia do estado (DCS, DCU, R30mm, Rx5dias e PRCPTOT), obtidos para as diferentes mesorregiões do Estado da Paraíba. Os índices foram correlacionados individualmente com a anomalia de TSM de cada área estudada e as correlações, com significância estatística ao nível de 95\%, estão apresentadas nas Tabelas 4 a 7. A significância estatística foi obtida através do teste $t$-Student aplicado a uma série de dados que apresenta graus de liberdade $(\mathrm{N})$ igual a 54 , correspondentes aos 54 anos da série histórica dos dados analisados.

\section{RESULTADOS E DISCUSSÃO}

\subsection{Tendências dos índices de extremos de precipitação para os períodos de 1935 a 1969 e 1970 a 2004}

As Tabelas 2 e 3 apresentam os valores das tendências dos índices de extremos de precipitação para os 23 postos

Tabela 2 - Tendência dos Índices de extremos climáticos na Paraíba para o período compreendido entre 1935 - 1969.

\begin{tabular}{|c|c|c|c|c|c|c|c|c|}
\hline Localidade & $\begin{array}{c}\text { PRCPTOT } \\
\text { (mm/ano) }\end{array}$ & $\begin{array}{c}\text { Rx1dia } \\
\text { (mm) }\end{array}$ & $\begin{array}{c}\text { Rx5dias } \\
\text { (mm) }\end{array}$ & $\begin{array}{r}\text { R10mm } \\
\text { (dia/ano) }\end{array}$ & $\begin{array}{r}\text { R30mm } \\
\text { (dia/ano) }\end{array}$ & $\begin{array}{c}\text { DCS } \\
\text { (dia/ano) }\end{array}$ & $\begin{array}{c}\text { DCU } \\
\text { (dia/ano) }\end{array}$ & $\begin{array}{c}\text { SDII } \\
\text { (mm/dia/ano) }\end{array}$ \\
\hline Aguiar & $-2,357$ & $-0,161$ & $-0,133$ & 0,017 & 0,025 & 0,918 & $-0,111$ & 0,170 \\
\hline Alhandra & 2,702 & 0,653 & $-0,422$ & 0,244 & 0,040 & 0,553 & $-0,083$ & 0,039 \\
\hline Areia & 3,000 & 0,219 & 0,089 & 0,020 & 0,012 & $-0,308$ & 0,090 & $-0,049$ \\
\hline Bananeiras & $-1,118$ & 0,251 & 0,213 & 0,013 & 0,148 & 1,203 & $-0,261$ & 0,361 \\
\hline B. de Santa Rosa & 2,260 & 0,474 & 0,371 & 0,122 & $-0,015$ & $-0,098$ & $-0,008$ & 0,070 \\
\hline Cabaceiras & 0,668 & 0,228 & $-0,112$ & 0,154 & 0,037 & 1,752 & $-0,095$ & 0,187 \\
\hline Catolé do Rocha & 1,247 & 0,301 & $-0,154$ & 0,066 & $-0,018$ & $-0,382$ & 0,067 & $-0,077$ \\
\hline Conceição & $-1,352$ & 0,603 & $-0,122$ & 0,104 & $-0,045$ & 1,248 & $-0,039$ & 0,121 \\
\hline Condado & 3,820 & $-0,601$ & $-0,343$ & 0,272 & 0,043 & $-1,053$ & 0,021 & $-0,010$ \\
\hline Imaculada & 4,208 & $-0,358$ & $-0,332$ & 0,129 & 0,055 & 0,749 & $-0,082$ & $-0,041$ \\
\hline Mamanguape & $-8,341$ & 0,356 & 0,530 & $-0,290$ & $-0,067$ & $-0,160$ & $-0,161$ & 0,084 \\
\hline Monteiro & 9,866 & 1,429 & 2,102 & 0,284 & 0,090 & $-2,248$ & 0,085 & $-0,125$ \\
\hline Nova Olinda & 8,710 & 0,801 & 1,781 & 0,258 & 0,131 & $-0,856$ & $-0,053$ & 0,327 \\
\hline Pedra Lavrada & 8,912 & 0,976 & 1,566 & 0,256 & 0,030 & 0,279 & 0,078 & 0,155 \\
\hline Pombal & 2,753 & 0,561 & 0,057 & 0,033 & 0,009 & $-0,289$ & 0,005 & $-0,082$ \\
\hline Santa Luzia & 0,518 & 0,310 & 0,369 & 0,012 & 0,003 & 0,252 & 0,039 & 0,015 \\
\hline São João do Cariri & $-0,741$ & 0,300 & 0,723 & $-0,028$ & 0,028 & 2,186 & $-0,050$ & 0,375 \\
\hline $\begin{array}{l}\text { S. J. do R. do } \\
\text { Peixe }\end{array}$ & 0,971 & 0,782 & 1,361 & $-0,079$ & $-0,019$ & 0,347 & $-0,039$ & 0,074 \\
\hline São João do Tigre & 15,459 & 1,129 & 3,131 & 0,343 & 0,147 & 0,036 & 0,090 & 0,146 \\
\hline Soledade & $-2,756$ & $-0,125$ & $-0,290$ & $-0,038$ & $-0,009$ & 1,820 & $-0,033$ & 0,082 \\
\hline Sousa & 3,813 & 0,458 & 0,414 & 0,151 & 0,033 & $-0,056$ & $-0,008$ & $-0,035$ \\
\hline Taperoá & 11,513 & 1,987 & 3,198 & 0,353 & 0,101 & $-2,865$ & 0,088 & 0,054 \\
\hline Teixeira & 5,307 & 0,759 & 0,416 & 0,230 & 0,031 & $-0,059$ & 0,049 & $-0,006$ \\
\hline
\end{tabular}

Valores realçados em cinza são tendências com nível de significância estatística de 5\%; Valores em negrito realçados em cinza são tendências com nível de significância de $1 \%$. 
Tabela 3 - Tendência dos índices de extremos climáticos na Paraíba para o período compreendido entre 1970 - 2004.

\begin{tabular}{lcccccccc}
\hline Localidade & $\begin{array}{c}\text { PRCPTOT } \\
\text { (mm/ano) }\end{array}$ & $\begin{array}{c}\text { Rx1dia } \\
(\mathbf{m m})\end{array}$ & $\begin{array}{c}\text { Rx5dias } \\
(\mathbf{m m})\end{array}$ & $\begin{array}{c}\mathbf{R 1 0 m m} \\
\text { (dia/ano) }\end{array}$ & $\begin{array}{c}\text { R30mm } \\
\text { (dia/ano) }\end{array}$ & $\begin{array}{c}\text { DCS } \\
\text { (dia/ano) }\end{array}$ & $\begin{array}{c}\text { DCU } \\
\text { (dia/ano) }\end{array}$ & $\begin{array}{c}\text { SDII } \\
\text { (mm/dia/ano) }\end{array}$ \\
\hline Aguiar & 1,679 & 0,066 & 0,342 & $-0,020$ & 0,054 & 1,257 & 0,030 & $-0,008$ \\
Alhandra & $-0,214$ & 1,097 & 1,059 & $-0,429$ & 0,135 & 0,235 & $-0,054$ & $-0,008$ \\
Areia & $-3,518$ & $-0,059$ & $\mathbf{- 0 , 8 1 5}$ & $-0,228$ & $-0,002$ & 0,018 & $-0,007$ & $-0,004$ \\
Bananeiras & 5,089 & $-0,651$ & $-0,859$ & 0,020 & 0,005 & $-0,428$ & 0,140 & $\mathbf{- 0 , 1 8 0}$ \\
Barra de Santa Rosa & 2,124 & $-0,077$ & $-0,235$ & $-0,035$ & $-0,004$ & $-1,634$ & 0,057 & $-0,105$ \\
Catolé do Rocha & $-3,687$ & 0,012 & $-0,164$ & $-0,136$ & $-0,030$ & $\mathbf{1 , 0 6 8}$ & 0,020 & 0,030 \\
Conceição & $-6,277$ & $-0,511$ & $-1,176$ & $-0,168$ & $-0,131$ & 0,579 & $\mathbf{0 , 0 2 5}$ & $-0,247$ \\
Condado & $-4,099$ & 0,412 & 0,059 & $-0,163$ & $-0,048$ & 1,874 & 0,010 & $-0,002$ \\
Imaculada & $-6,279$ & 0,129 & $-0,961$ & $-0,222$ & $-0,080$ & $\mathbf{0 , 9 1 8}$ & $-0,016$ & $-0,084$ \\
Mamanguape & $-9,459$ & 0,952 & $-0,810$ & $-0,598$ & 0,034 & 0,467 & $-0,082$ & $-0,092$ \\
Monteiro & $-0,715$ & 0,395 & 0,123 & $-0,022$ & $-0,007$ & $-1,063$ & 0,010 & 0,061 \\
Nova Olinda & $-22,204$ & $\mathbf{- 1 , 3 6 9}$ & $-3,063$ & $-0,507$ & $\mathbf{- 0 , 1 3 4}$ & 0,786 & $-0,022$ & $-0,332$ \\
Pedra Lavrada & $-2,574$ & $-0,195$ & 0,037 & $-0,015$ & $-0,016$ & 2,466 & $-0,105$ & 0,195 \\
Pombal & 2,119 & 1,188 & 1,925 & 0,021 & $-0,024$ & 0,197 & 0,108 & $-\mathbf{0 , 0 5 5}$ \\
Santa Luzia & $-7,943$ & $-0,618$ & $-1,462$ & $\mathbf{- 0 , 2 3 5}$ & $-0,097$ & 0,500 & $-0,017$ & $-0,085$ \\
São João do Cariri & 2,505 & $-0,001$ & $-0,287$ & 0,048 & $-0,004$ & $-0,294$ & 0,024 & $-\mathbf{0 , 2 2 5}$ \\
S. J. do R. do Peixe & $-0,502$ & 0,121 & $-0,025$ & $-0,053$ & 0,056 & 2,088 & $-0,044$ & 0,177 \\
São João do Tigre & $-8,760$ & $-0,473$ & $-0,606$ & $-0,353$ & $-0,060$ & 0,668 & $-0,065$ & $-0,042$ \\
Soledade & $-3,363$ & $-0,335$ & $-1,652$ & $-0,107$ & $-0,051$ & $-0,643$ & $-0,026$ & $-0,173$ \\
Sousa & $-2,909$ & $\mathbf{- 0 , 8 8 9}$ & $-1,187$ & $-0,052$ & $-0,058$ & 0,089 & $-0,017$ & $-0,059$ \\
Taperoán & $-2,494$ & 0,477 & $-0,428$ & $-0,122$ & $-0,023$ & 1,685 & $\mathbf{- 0 , 0 3 9}$ & 0,032 \\
Teixeira & 0,038 & 0,131 & 0,061 & 0,017 & $-0,071$ & $-1,793$ & 0,110 & $-0,290$ \\
\hline
\end{tabular}

Valores realçados em cinza são tendências com nível de significância estatística de 5\%;

Valores em negrito realçados em cinza são tendências com nível de significância de 1\%.

pluviométricos na Paraíba, para os períodos de 1935 a 1969 e de 1970 a 2004, respectivamente. Na Tabela 2 é possível observar que das 17 localidades que apresentaram tendência de aumento na precipitação total anual (PRCPTOT), apenas 4 mostraram significância estatística com nível de significância de $5 \%(\mathrm{p} \leq 0,05)$. As referidas localidades foram Nova Olinda, Pedra Lavrada, São João do Tigre e Taperoá, onde três destas localidades estão inseridas na Mesorregião da Borborema e uma no Sertão (Nova Olinda) (Figura 2a). Ainda na Tabela 2 , identifica-se que das seis localidades que apresentaram tendências de decréscimo do PRCPTOT, nenhuma apresentou significância estatística para os níveis adotados. Assim, durante o primeiro período analisado (1935 a 1969) foram observadas tendências predominantes de aumento na precipitação total anual sobre a Paraíba, especialmente na Mesorregião da Borborema.

Por outro lado, observa-se na Tabela 3 que 6 localidades apresentaram tendências de aumento no índice PRCPTOT, porém nenhuma destas com significância estatística e, das 17 localidades com tendências de decréscimo no referido índice, apenas 2 têm significância estatística (Nova Olinda e São João do Tigre) (Figura 2a) e estão localizadas na parte sul do estado nas Mesorregiões do Sertão e Borborema, respectivamente. É interessante notar que essas localidades apresentaram tendências de aumento para o PRCPTOT no período de 1935 a 1969 e de diminuição no período de 1970 a 2004, ambas com significância estatística, caracterizando que durante os últimos anos, a precipitação total sobre essas localidades tem sido reduzida consideravelmente, ou seja, mostraram tendências de sinais contrários para os dois períodos analisados. Estes resultados concordam parcialmente com os apresentados por Haylock et al. (2006) e Santos e Brito (2007). Entretanto, a coerência espacial dos índices de precipitação é muito baixa. O padrão espacial de tendências positivas e negativas é muito misturado mesmo quando estações individuais são analisadas, corroborando assim, com os resultados encontrados por Aguilar et al. (2009).

Tendências semelhantes às obtidas para o PRCPTOT, entre os dois períodos analisados, também foram encontradas para os demais índices. A propósito o Rx1dia, mostrado na Tabela 2 (período de 1935 a 1969), apresentou tendências de 
a)

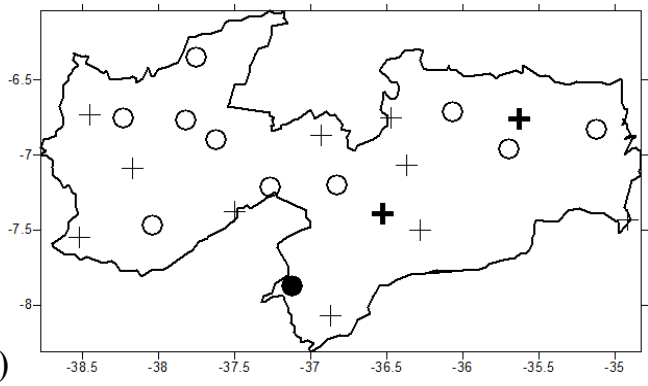

c)

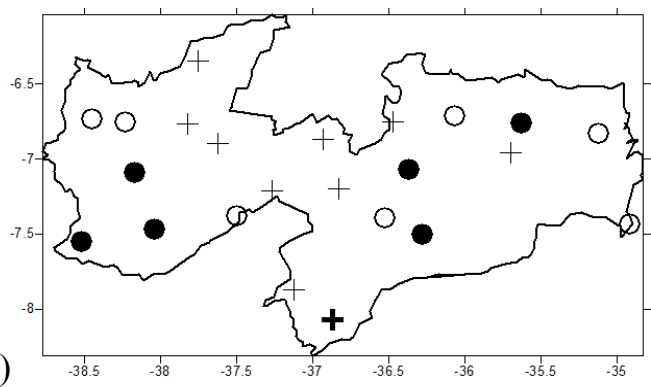

e)

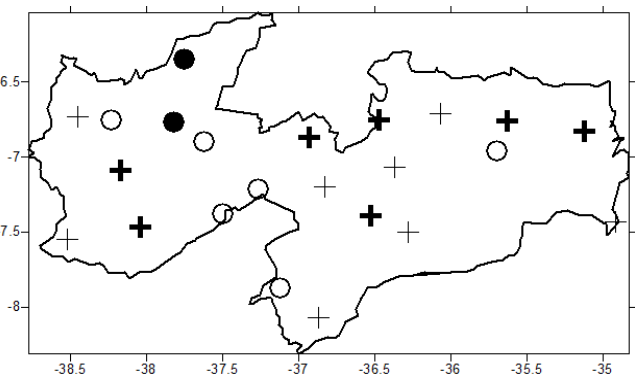

g)

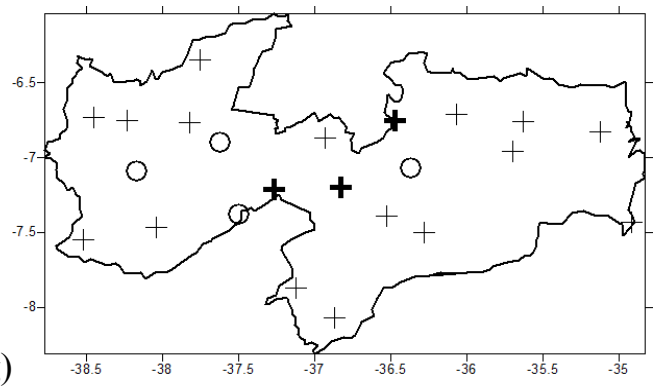

i)

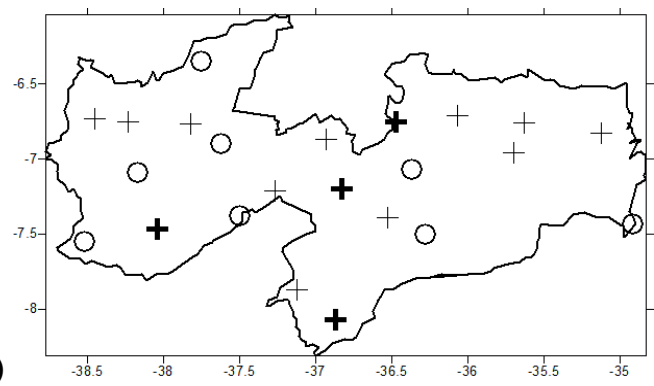

b)

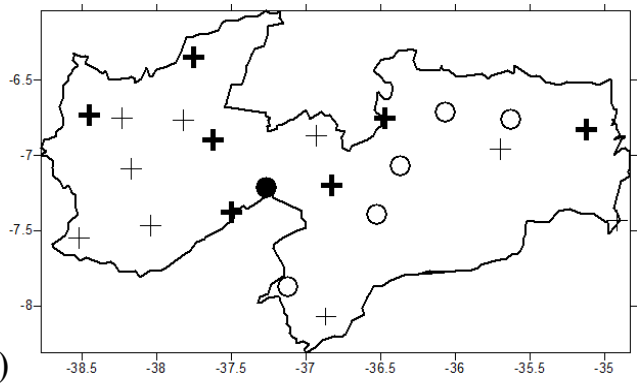

d)

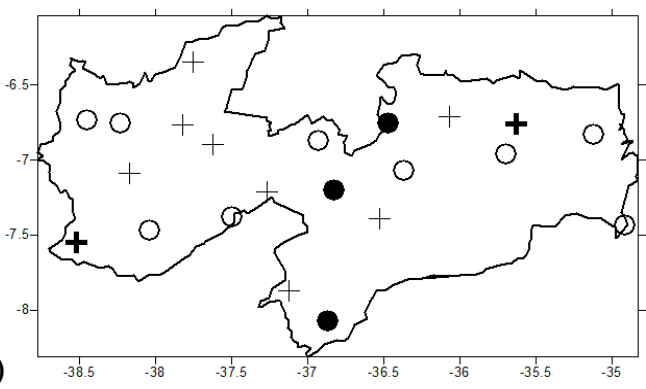

f)

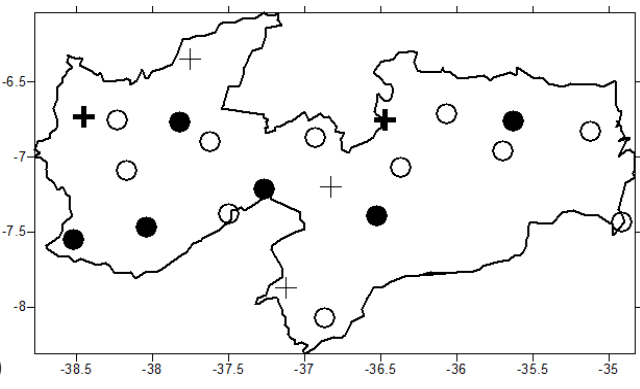

h)

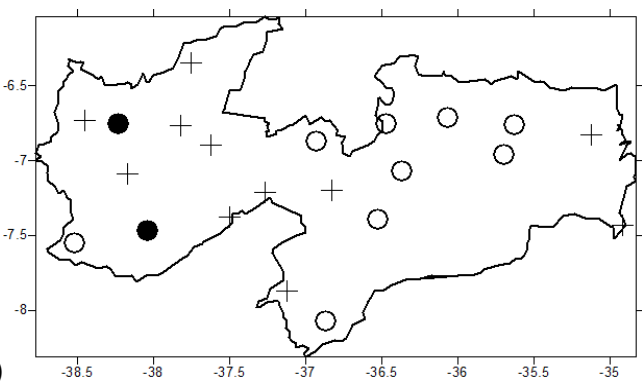

j)

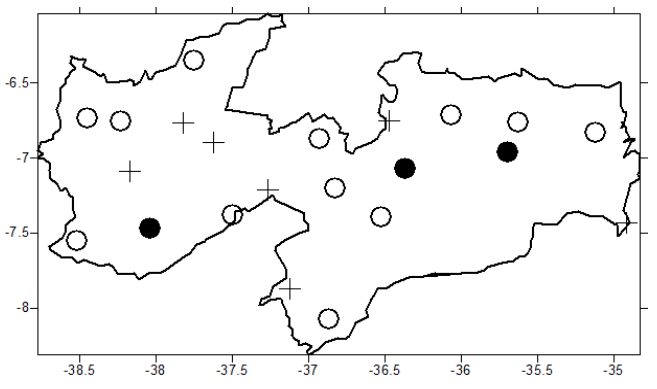

Figura 2 - Distribuição espacial das tendências dos índices de extremos climáticos para o estado da Paraíba onde, a) DCS, c) DCU, e) SDII, g) Rx1day, i) Rx5day, k) PRCPTOT, m) R10 mm e o) R30 mm no período de (1935 a 1969); b) DCS, d) DCU, f) SDII, h) Rx1day, j) Rx5day, l) PRCPTOT, n) R10 mm e p) R30 mm no período de (1970 a 2004). Continua 
$\mathrm{k})$

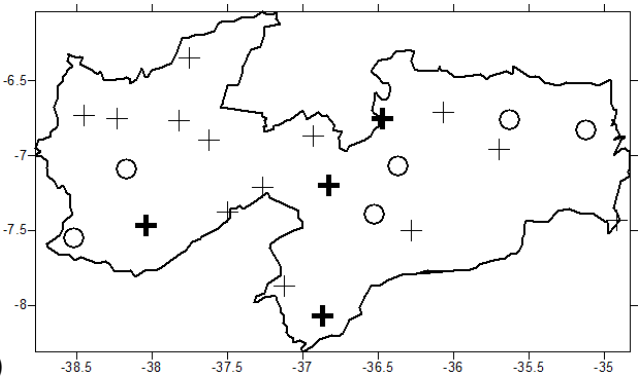

$\mathrm{m})$

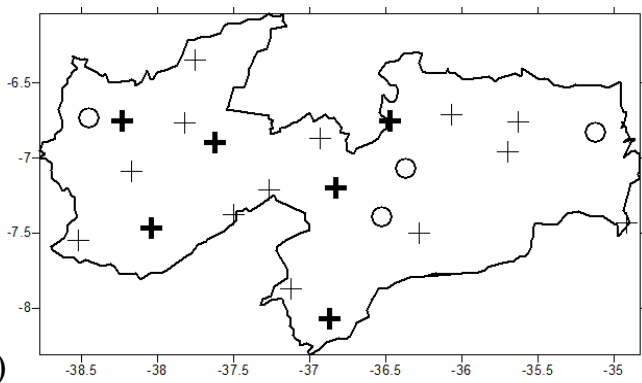

o)

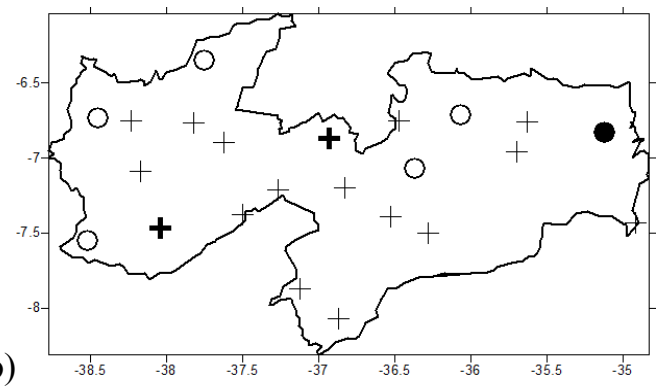

1)

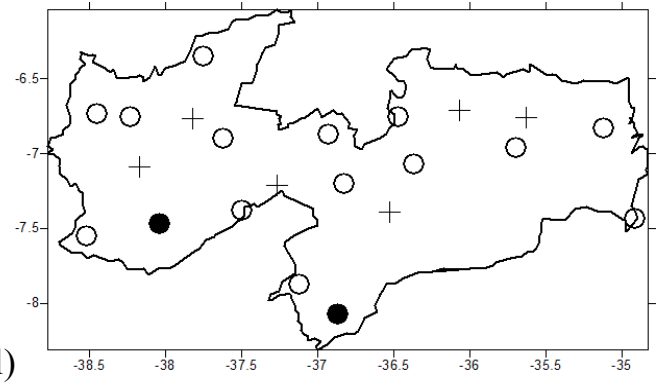

n)

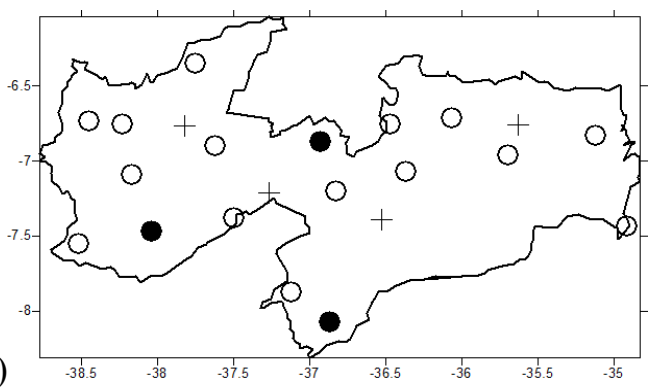

p)

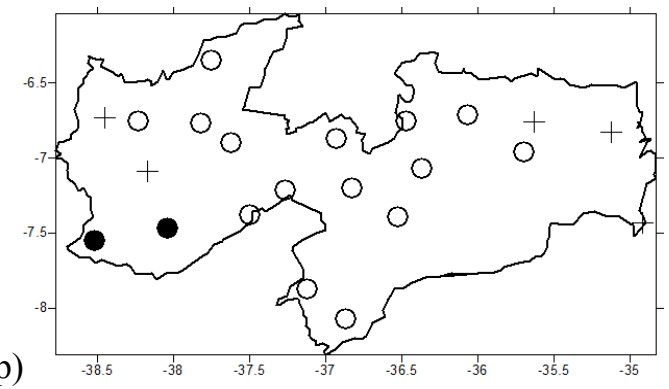

Figura 2 - Conclusão

aumento significativo em 3 localidades (Pedra Lavrada, Taperóa e Teixeira) e nenhuma com tendência significativa de decréscimo na precipitação máxima em um único dia (Figura 2c). Esse resultado evidencia que nas referidas localidades, durante o primeiro período estudado, os eventos de precipitação isolados, ou seja, em um único dia, foram intensificados e contribuíram para o aumento na precipitação total anual, com destaque para Pedra Lavrada que apresentou aumento da PRCPTOT e de Rx1dia. Na Tabela 3 estão apresentadas as tendências do índice Rx1dia para o período de 1970 a 2004, onde pode-se notar que nenhuma localidade apresentou tendência de aumento com significância e apenas 2 com tendências de decréscimo com significância (Nova Olinda e Sousa) (Figura 2d). Vale salientar que Nova Olinda também apresentou decréscimo na PRCPTOT para esse período, indicando que devido à redução dos eventos isolados de precipitação, caracterizado pelo índice Rx1dia, a precipitação total anual também foi reduzida. No primeiro período estudado, as tendências estatisticamente significativas se encontram, essencialmente, na Mesorregião da Borborema, enquanto que no segundo período, estão dispostas no Sertão.
Análise similar à anteriormente apresentada pode ser feita para o índice Rx5dias, mostrado na Tabela 2 para o período de 1935 a 1969. O referido índice apresentou tendências de aumento significativo em 4 localidades (Nova Olinda, Pedra Lavrada, São João do Tigre e Taperóa) e nenhuma com tendência significativa de decréscimo na precipitação máxima em cinco dias consecutivos (Figura 2e). Esse resultado indica que nestas localidades, em especial Pedra Lavrada, entre 1935 e 1969, os eventos de precipitação em cinco dias consecutivos foram intensificados, contribuindo para o aumento na precipitação total anual. Na Tabela 3 também estão apresentadas as tendências do índice Rx5dias para o período de 1970 a 2004, e verifica-se que nenhuma das localidades apresentou tendência de aumento com significância e apenas 3 com tendências de decréscimo com significância (Areia, Nova Olinda e Soledade) (Figura 2f). Mais uma vez, salienta-se que Nova Olinda também apresentou decréscimo nos índices PRCPTOT e Rx1dia para esse período, indicando que devido à redução dos eventos de precipitação em 1 e 5 dias consecutivos, caracterizados pelos índices Rx1dia e 
Rx5dias, a precipitação total anual foi reduzida, conforme discussão anterior.

Nota-se na Tabela 2, que o índice R10mm apresentou 6 localidades com tendência significativa de aumento, ou seja, as localidades Condado, Nova Olinda, Pedra Lavrada, São João do Tigre, Sousa e Taperoá tiveram aumento das chuva iguais ou superiores a $10 \mathrm{~mm}$ para o período de 1935 a 1969 (Figura 2g), enquanto que, as localidades Nova Olinda, Santa Luzia e São João do Tigre mostraram tendências significativas de diminuição das chuvas iguais ou superiores a $10 \mathrm{~mm}$ nas últimas décadas (Figura $2 \mathrm{~h}$ e Tabela 3). Mais uma vez, Nova Olinda se apresenta como uma localidade em que o padrão de precipitação tem se alterado, quando comparados os dois períodos estudados. Quanto ao índice R30mm, que representa o número de casos anuais em que a precipitação foi igual ou superior a $30 \mathrm{~mm}$, este mostrou uma localidade com tendência significativa de decréscimo (Mamanguape) e 2 com tendências de aumento significativo (Nova Olinda e Santa Luzia) durante o primeiro período estudado (Figura 2i). Para o segundo período, foram observadas apenas tendências significativas de decréscimo para 2 localidades (Conceição e Nova Olinda) (Figura 2j). Esses resultados indicam, mais uma vez, que Nova Olinda apresentou alteração no seu padrão de precipitação nas últimas décadas. É interessante notar que as tendências estatisticamente significativas estão dispostas, essencialmente, nas Mesorregiões da Borborema e Sertão, que são áreas de clima semiárido e com vegetação de Caatinga.

Os índices DCS e DCU apresentaram tendências significativas de aumento e de decréscimos em ambos os períodos estudados. Na Tabela 2 é possível identificar que o DCS foi positivo em 2 localidades (Bananeiras e São João do Cariri) e negativo em 1 (Monteiro) (Figura 2k) para o período de 1935 a 1969, enquanto que na Tabela 3, o referido índice apresentou tendências positivas para 7 localidades e negativa para 1 (Teixeira) (Figura 21). Esses resultados indicam que durante as últimas décadas houve aumento predominante do número de dias consecutivos secos nas diferentes Mesorregiões da Paraíba, o que corrobora com a redução da precipitação total anual. O índice DCU (Tabela 2) apresenta 6 localidades com tendências significativas de decréscimo e uma (São João do Tigre) com tendência de aumento (Figura $2 \mathrm{~m}$ ). Na Tabela 3, o referido índice mostra tendências significativas de decréscimo em 3 localidades (Pedra Lavrada, São João do Tigre e Taperóa) e tendências de aumento para duas (Bananeiras e Conceição) (Figura 2n). Assim, esses resultados não deixam claro o comportamento dos dias consecutivos úmidos sobre as Mesorregiões da Paraíba, pois apresentam tendências de sinais contrários com significância estatística, para o mesmo período analisado. Entretanto, as localidades de Bananeiras e Conceição mostram resultados opostos para os dois períodos, indicando que as mesmas tiveram reduções no número de dias consecutivos anuais com precipitação pluviométrica durante as últimas décadas.

Baseado nos resultados apresentados pelos índices de extremos climáticos, oriundos da precipitação pluvial diária, é possível conjecturar que a intensificação do uso da vegetação e do solo, levando a fortes mudanças nas características da ocupação da terra, acarretando na degradação dos ecossistemas naturais (vegetação da região semiárida), tem causado influência na distribuição espaço-temporal da precipitação total anual e de seus extremos sobre a Paraíba. Oyama e Nobre (2003) mostraram que as mudanças de uso da terra ocasionam profundos impactos no ciclo hidrológico, tanto na Amazônia, como no Nordeste do Brasil. Vale salientar que no ano de 2002 a Paraíba assumiu o primeiro lugar no ranking dos Estados que mais devastavam o meio ambiente. O Instituto Brasileiro do Meio Ambiente e dos Recursos Naturais Renováveis (IBAMA) estimou que cerca de 17 mil hectares de lenha eram retirados das matas paraibanas. Corroborando com o Ibama, Schenkel e Matallo Júnior (2003), em seu relatório "Mudanças de clima, mudanças de vida", indicam que $29 \%$ do território paraibano estão comprometidos, afetando diretamente cerca de 653 mil pessoas. Pode-se destacar, também, que as principais causas da degradação dos solos na Paraíba ocorrem devidas ao uso inadequado dos recursos da terra, que associados com as secas e ação antrópica desordenada podem causar danos irreversíveis. Os usos do solo mais nocivos ao ambiente na Paraíba são cultivo em terras inapropriadas, pecuária extensiva e desmatamento das vegetações nativas e das margens de rios.

Os resultados apresentados nas Tabelas 2 e 3 mostram que, de um modo geral, no período de 1935 a 1969 verificou-se uma tendência de aumento da precipitação na Paraíba, enquanto no de 1970 a 2004 ocorreu uma tendência de diminuição. As causas para que isto tenha ocorrido pode ser tanto de grande escala como de escala regional, principalmente esta última, uma vez que as atividades antrópicas no Nordeste do Brasil, e em particular na Paraíba, foram intensificadas nos últimos trinta anos do século passado. A propósito, informações do Banco de Dados do Instituto Brasileiro de Geografia e Estatística (IBGE, 1997) mostram que no ano de 1977 a área plantada por cultura agrícola na Paraíba foi de aproximadamente 1,6 milhões de hectare. Entretanto, outros setores econômicos do Estado também produzem desmatamento. De acordo com Silva (1994), no ano de 1993 foram retirando mais de 2,7 milhões de metro cúbico de lenha das áreas vegetadas da Paraíba apenas para o consumo de energia, tanto residencial, como comercial e industrial.

Como uma área desmatada leva um tempo relativamente grande para se restabelecer, mesmo que não esteja mais recebendo pressões antrópicas, pode-se afirmar que uma das 
possíveis causas para uma tendência de diminuição das chuvas no estado da Paraíba no período de 1970 a 2004 são as atividades antrópicas, principalmente o desmatamento.

\subsection{Correlações entre os índices de extremos de precipitação e anomalias de TSM dos Oceanos Tropicais}

É observado através da Tabela 4, que o índice DCS tem correlação positiva estatisticamente significativa ao nível de $5 \%$ com as anomalias de TSM indicando que quando as águas do Atlântico Norte (TNAI) estão mais quentes, ocorre um aumento na quantidade de dias consecutivos secos. De acordo com as correlações negativas de TNAI e OMA com R30mm e PRCPTOT há uma diminuição das chuvas de $30 \mathrm{~mm}$ e da precipitação total no Sertão da Paraíba. Assim sendo, quando as águas no Atlântico Sul (TSAI) estão mais aquecidas que a média climatológica, a Zona de Convergência Intertropical (ZCIT) se posiciona mais ao sul (Chung, 1982), tem-se uma redução de DCS e um aumento de PRCPTOT. Esses resultados concordam com Moura et al. (2009) que encontraram as maiores correlações de precipitação no leste do NEB com as TSM do Atlântico. Na mesma Tabela 4, as correlações negativas (com significância estatística ao nível de 5\%) dos índices R30mm, Rx5day e PRCPTOT com as anomalias nas regiões de Niño $1+$ 2 e Niño 3, no Oceano Pacífico, indicam que anomalias positivas da TSM dessas regiões contribuem para reduzir as chuvas mais intensas (iguais ou superiores a $30 \mathrm{~mm}$ ), o acumulado de 5 dias consecutivos e na precipitação total no Sertão.

Através da Tabela 5 verifica-se uma correlação positiva com significância estatística de 5\% entre DCU e ODP, e uma correlação negativa mais fraca entre DCS e ODP, indicando que esse modo de variabilidade de temperatura no Pacífico influencia nos dias consecutivos secos e úmidos na mesorregião da Borborema. Similarmente, as correlações entre os índices DCS e DCU e a OMA indicam que na sua fase quente (fria) há um aumento (diminuição) de dias secos e consequentemente, diminuição (aumento) de dias úmidos nessa região do Estado. De acordo ainda com as correlações negativas de R30mm, Rx5day e PRCPTOT com OMA e TNAI, pode-se ver que quando nessas áreas do Atlântico as superfícies do mar estão mais aquecidas que a média climatológica, há uma redução desses índices extremos de precipitação sobre a Mesorregião da Borborema. Isso indica que quando as águas na parte norte do Atlântico (TNAI) estão mais frias, a ZCIT é deslocada para o sul de sua posição predominante, influenciando assim, positivamente no regime chuvoso no NEB (Santos, 2006). Não se verifica nenhuma correlação ao nível de $5 \%$ dos índices de precipitação com Niño $1+2$ e Niño 3 no Pacífico.

Os dias consecutivos secos no Agreste da Paraíba têm correlação negativa estatisticamente significativa com a ODP (Tabela 6), mostrando que a sua fase quente favorece a redução

Tabela 4 - Correlações entre anomalias de TSM e os índices extremos de precipitação para a Mesorregião do Sertão na Paraíba (1950 a 2004).

\begin{tabular}{llllll}
\hline & DCS & DCU & R30mm & Rx5day & PRCPTOT \\
\hline ODP & $-0,080$ & 0,182 & $-0,122$ & $-0,059$ & $-0,090$ \\
OMA & $0,401^{* *}$ & $-0,066$ & $-0,457^{* *}$ & $-0,223$ & $-0,512^{* *}$ \\
TNAI & $0,296^{*}$ & $-0,058$ & $-0,513^{* *}$ & $-0,194$ & $-0,528^{* *}$ \\
TSAI & $-0,281^{*}$ & 0,158 & 0,167 & $-0,045$ & 0,201 \\
NIÑO 1+2 & $-0,019$ & $-0,138$ & $-0,368^{* *}$ & $-0,308^{* *}$ & $-0,366^{* *}$ \\
NIÑO 3 & 0,028 & $-0,103$ & $-0,355^{* *}$ & $-0,257^{*}$ & $-0,356^{* *}$ \\
\hline
\end{tabular}

* significativo para o nível de $5 \%$ (quando rCal $\geq$ rcrit) rcrit $\geq 0,254$

** significativo para o nível de $1 \%$ (quando rcrit $\geq 0,300$ )

Tabela 5 - Correlações entre anomalias de TSM e os índices extremos de precipitação para a Mesorregião da Borborema na Paraíba (1950 a 2004$).$

\begin{tabular}{llllll}
\hline & DCS & DCU & R30mm & Rx5day & PRCPTOT \\
\hline ODP & $-0,207$ & $0,281^{*}$ & $-0,015$ & 0,178 & 0,027 \\
OMA & $0,357^{* *}$ & $-0,284^{*}$ & $-0,462^{* *}$ & $-0,377^{* *}$ & $-0,545^{* *}$ \\
TNAI & 0,179 & $-0,173$ & $-0,500^{* *}$ & $-0,355^{* *}$ & $-0,519^{* *}$ \\
TSAI & $-0,105$ & 0,118 & 0,107 & 0,087 & 0,188 \\
NIÑO 1+2 & 0,039 & $-0,042$ & $-0,172$ & $-0,037$ & $-0,230$ \\
NIÑO 3 & 0,036 & $-0,049$ & $-0,206$ & $-0,055$ & $-0,232$ \\
\hline
\end{tabular}

* significativo para o nível de $5 \%$ (quando rCal $\geq$ rcrit) rcrit $\geq 0,254$

** significativo para o nível de $1 \%$ (quando rcrit $\geq 0,300$ ) 
dos dias consecutivos secos. Concordando com os resultados e discussão anteriores, as correlações negativas dos índices R30mm, Rx5day e PRCPTOT com TNAI e OMA apontam para redução desses índices no Agreste, ou seja, quando a OMA está na sua fase fria e TNAI mais fria, a ZCIT fica mais ao sul e as chuvas nessa Mesorregião são influenciadas positivamente. Já o Pacífico contribui para chuvas nessa Região, quando as águas superficiais estão mais frias que média (La Niña) nas regiões de Niño $1+2$ e Niño 3, apresentado pelas correlações negativas (Tabela 6) entre os índices R30mm e Rx5day com Niño $1+2$ e Niño 3 que mostra que a precipitação total anual é fortemente dependente das chuvas acima de $30 \mathrm{~mm}$ e do acumulado em 5 dias consecutivos.

A Mesorregião do Litoral da Paraíba tem um comportamento influenciado devido ao Dipolo do Atlântico, como indicado pelos índices DCS, DCU e PRCPTOT, os quais apresentam correlações opostas com TNAI e TSAI (Tabela 7), indicando que quando a TSM do Atlântico norte está mais quente (fria), há um deslocamento da ZCIT para o norte (sul) de sua posição predominante, desfavorecendo (favorecendo) a formação de chuvas sobre o NEB, e o número de dias consecutivos secos aumenta (diminui), com também, os dias consecutivos úmidos a precipitação total diminuem (aumentam). Os índices de extremos climáticos não apresentaram correlação com a TSM do Pacífico, e nem com a ODP e a OMA. Portanto, o comportamento da variabilidade temporal da precipitação sobre o Litoral está mais associado às brisas marítimas, linhas de instabilidade e sistemas de leste.

\section{CONCLUSÕES}

Com base nos resultados apresentados anteriormente, é possível concluir que o período de 1935 a 1969 apresentou tendência predominante de aumento da precipitação na Paraíba, enquanto no de 1970 a 2004 ocorreu uma tendência de redução das precipitações. Devido à variabilidade espacial conjectura-se que as causas para essa modificação pode ser tanto de grande escala, como de escala regional, haja vista que a Paraíba, nos últimos trinta anos do século passado, apresentou intensificação das atividades antrópicas.

No primeiro período estudado, as tendências estatisticamente significativas foram, predominantemente, na Mesorregião da Borborema, enquanto que no segundo período, estão dispostas no Sertão.

Também se observou que os índices de eventos extremos de precipitação no Sertão da Paraíba estão mais associados com as anomalias de TSM dos Oceanos, Atlântico e Pacífico, como também, das Oscilações Multidecadal do Atlântico. Já os índices de eventos extremos de precipitação na Borborema e no Agreste são mais correlacionados com as TSM do Atlântico na região de TNAI e da OMA. Entretanto, para o Litoral paraibano, os índices extremos de precipitação não apresentaram relação com

Tabela 6 - Correlações entre anomalias de TSM e os índices extremos de precipitação para a Mesorregião do Agreste na Paraíba (1950 a 2004).

\begin{tabular}{llllll}
\hline & DCS & DCU & R30mm & Rx5day & PRCPTOT \\
\hline ODP & $-0,278^{*}$ & 0,063 & $-0,013$ & 0,108 & 0,096 \\
OMA & 0,184 & 0,118 & $-0,181$ & $-0,314^{* *}$ & $-0,342^{* *}$ \\
TNAI & 0,034 & 0,076 & $-0,336^{* *}$ & $-0,269^{*}$ & $-0,384^{* *}$ \\
TSAI & $-0,273^{*}$ & $-0,110$ & 0,056 & $-0,037$ & 0,154 \\
NIÑO 1+2 & $-0,076$ & $-0,071$ & $-0,296^{*}$ & $-0,318^{* *}$ & $-0,199$ \\
NIÑO 3 & $-0,116$ & $-0,013$ & $-0,282^{*}$ & $-0,260^{*}$ & $-0,166$ \\
* significativo para o nível de 5\% (quando rCal $\geq$ rcrit) rcrit $\geq 0,254$ \\
** significativo para o nível de 1\% (quando rcrit $\geq 0,300$ )
\end{tabular}

Tabela 7 - Correlações entre anomalias de TSM e os índices extremos de precipitação para a Mesorregião do Litoral na Paraíba (1950 a 2004).

\begin{tabular}{llllll}
\hline & DCS & DCU & R30mm & Rx5day & PRCPTOT \\
\hline ODP & 0,118 & 0,192 & 0,003 & 0,034 & $-0,004$ \\
OMA & 0,242 & $-0,115$ & $-0,118$ & $-0,059$ & $-0,222$ \\
TNAI & $0,271^{*}$ & $-0,276^{*}$ & $-0,174$ & $-0,036$ & $-0,298^{*}$ \\
TSAI & 0,076 & 0,002 & 0,202 & 0,047 & 0,247 \\
NIÑO 1+2 & 0,011 & $-0,013$ & $-0,204$ & $-0,248$ & $-0,212$ \\
NIÑO 3 & 0,026 & 0,111 & $-0,174$ & $-0,193$ & $-0,151$ \\
\hline
\end{tabular}

* significativo para o nível de 5\% (quando rCal $\geq$ rcrit) rerit $\geq 0,254$

** significativo para o nível de $1 \%$ (quando rcrit $\geq 0,300$ ) 
o Pacífico, e sim, com o Atlântico (TNAI), como encontrou Moura et al. (2009) para o leste do Nordeste do Brasil. A mesma, pode também ser mais dependentes das brisas marítimas, linhas de instabilidade e sistemas de leste.

A forte relação entre os padrões de precipitação do Nordeste do Brasil com os Oceanos Atlântico e Pacífico já é vastamente conhecida da literatura (Nobre e Shukla, 1996; Hastenrath, 2000a; 2000b; 2006; Santos e Brito, 2007). No entanto, as constatações dos estudos anteriores referiam-se aos padrões de precipitação mais gerais. A constatação das correlações entre índices indicadores de alterações desses padrões e o comportamento térmico dos Oceanos confirmam o quão são minuciosas e complexas essas relações.

\section{REFERÊNCIAS BIBLIOGRÁFICAS}

AGUILAR, E.; AZIZ BARRY, A.; BRUNET, M.; EKANG, L.; FERNANDES, A.; MASSOUKINA, M.; MBAH, J.; MHANDA, A.; DO NASCIMENTO, D.J.; PETERSON, T.C.; THAMBA UMBA, O.; TOMOU, M.; ZHANG, X. Changes in temperature and precipitation extremes in western central Africa, Guinea Conakry, and Zimbabwe, 1955-2006. Journal of Geophysical Research, v. 114, d02115, 2009.

CHUNG, J. C. Correlations between the tropical Atlantic trade winds and precipitation in Northeastern Brazil. Journal of Climatology, v.2, p. 35-46, 1982.

HASTENRATH, S. Circulation and teleconnection mechanisms of Northeast Brazil droughts. Progress in Oceanography, v.70, p.407-415, 2006.

HASTENRATH, S. Exploring the climate problems of Brazil's Nordeste: a review. Climatic Change, v. 112, p.243-251, 2012.

HASTENRATH, S. Interannual and longer-term variability of upper air circulation in the Northeast Brazil-tropical sector. Journal of Geophysical Research-Atmospheres, v. 105, p.7327-7335, 2000a.

HASTENRATH, S. Upper air mechanisms of the Southern Oscillation in the tropical Atlantic sector. Journal of Geophysical Research-Atmosphere, v. 105, p.1499715009, 2000b.

HAYLOCK, M.R.; PETERSON, T.C.; ALVES, L.M.; AMBRIZZI, T.; ANUNCIAÇÃO, Y.M.T.; BAEZ, J.; BARROS, V.R.; BERLATO, M.A.; BIDEGAIN, M.; CORONEL, G.; GARCIA, V.J.; GRIMM, A.M.; KAROLY, D.; MARENGO, J.A.; MARINO, M.B.; MONCUNILL, D.F.; NECHET, D.; QUINTANA, J.; REBELLO, E.; RUSTICUCCI, M.; SANTOS, J.L.; TREBEJO, I.; VINCENT, L.A. Trends in total and extreme South American rainfall 1960-2000 and links with sea surface temperature. Journal of Climate, v. 19, p.1490-1512, 2006.
IBGE. Produção agrícola municipal 1975-1994. IBGE, Departamento de agropecuária. Rio de Janeiro: IBGE, 1997. 726p. (ISBN 85-2400644-7).

INTERGOVERNMENTAL PANEL ON CLIMATE CHANGE (IPCC). Climate Change 2007 - The Physical Science Basis. Contribution of Working Group I to the Fourth Assessment Report of the IPCC. Cambridge University Press, Cambridge, 2007.

KOSTOPOUlO, E., JONES, P. D. Assessment of climate extremes in the Eastern Mediterranean. Meteorology and Atmospheric Physics, v. 89, p. 69-85, 2005.

MENEZES, H. E. A.; BRITO, J. I. B.; SANTOS, C. A. C.; SILVA, L. L. A relação entre a temperatura da superfície dos oceanos tropicais e a duração dos veranicos no estado da Paraíba. Revista Brasileira de Meteorologia, v. 23, p.152-161, 2008. MOURA, G. B. A.; ARAGÃO, J. O. R.; MELO, J. S. P.; SILVA, A. P. N.; GIONGO, P. R.; LACERDA, F. F. Relação entre a precipitação do leste do Nordeste do Brasil e a temperatura dos oceanos. Revista Brasileira de Engenharia Agrícola e Ambiental, v. 13, p. 462-469, 2009.

NOBRE, P., SHUKLA, J. Variations of sea surface temperature, wind stress and rainfall over the tropical Atlantic and South America. Journal of Climate, v.10, p.2464-2479, 1996.

OYAMA, M. D.; NOBRE, C. A. A new climate-vegetation equilibrium state for Tropical South America. Geophysical Research Letters, v.30, 2199, 2003

SANTOS, C. A. C. Estimativas e tendências de índices de detecção de mudanças climáticas com base na precipitação diária no Rio Grande do Norte e na Paraíba. Dissertação de Mestrado em Meteorologia, Universidade Federal de Campina Grande, Unidade Acadêmica de Ciências Atmosféricas. Campina Grande, 115 p, 2006.

SANTOS, C. A. C.; BRITO, J. I. B. Análise dos índices de extremos para o semi-árido do Brasil e suas relações com TSM e IVDN. Revista Brasileira de Meteorologia, v.22, p.303-312, 2007.

SANTOS, C. A. C.; BRITO, J. I. B.; RAO, T. V. R.; MENEZES, H. E. A. Tendência dos Índices de Precipitação no Estado do Ceará. Revista Brasileira de Meteorologia, v.24, p.3947, 2009.

SANTOS, C. A. C.; NEALE, C. M. U.; SILVA, B. B.; RAO, T. V. R. Trends in indices for extremes in daily temperature and precipitation over Utah, USA. International Journal of Climatology, v. 31, p.1813-1822, 2011.

SANTOS, C. A.; MANZI. A. O. Eventos extremos de precipitação no estado do Ceará e suas relações com a temperatura dos Oceanos Tropicais. Revista Brasileira de Meteorologia, v.26, p.157-165, 2011.

SCHENKEL, C. S.; MATALLO JÚNIOR, H. (Org.) Desertificação. 2. ed. Brasília: UNESCO, 2003. 82p. 
SILVA, J. A. Avaliação do estoque lenhoso - inventário florestal do estado da Paraíba. João Pessoa: Projeto PNUD/FAO/IBAMA - Governo da Paraíba, 1994. 27p. (Documento de Campo 21).

SNEYERS, R. On the Statistical Analysis of Series of Observations. Technical Note No. 143, WMO No. 415 World Meteorological Organization: Geneva. 1990.

VINEY, N. R.; BATES, B. C. It never rains on Sunday: The prevalence and implications of untagged multi-day rainfall accumulations in the Australian high quality data set. International Journal of Climatology, v. 24, p.1171-1192, 2004.
YOU, Q.; KANG, S.; AGUILAR, E.; PEPIN, N.; FLUGEL, W.; YAN, Y.; XU, Y.; ZHANG, Y.; HUANG, J. Changes in daily climate extremes in China and their connection to the large scale atmospheric circulation during 1961-2003. Climate Dynamics, v. 36, p. 2399-2417, 2010.

ZHANG, X.; HEGERL, G.; ZWIERS, F.; KENYON, J. Avoiding inhomogeneity in percentile-based indices of temperature extremes. Journal of Climate, v. 18, p.1641-1651, 2005.

ZHANG, X.; YANG, F. RClimDex (1.0) User Guide. Climate Research Branch Environment Canada: Downsview, Ontario, Canada, 2004.f 